\title{
Fermented products in nutrition
}

\begin{abstract}
Most nutritional guidelines in the world, including in Asian countries where fermented foods are widely used, do not mention fermented foods and beverages besides yoghurt as a dairy product. Children get their gut micro biota from the stage of birth and milking and the intestinal flora remains most the same throughout their life. Fermented foods and mushroom biomass may modulate the pro biotic profile of gut flora and thereby the immune system.
\end{abstract}

Volume 8 Issue 2 - 2018

\author{
Victoria Bell,' Jorge Ferrão, ${ }^{2}$ Tito Fernandes ${ }^{3}$ \\ 'Faculty of Pharmacy Coimbra University, Portugal \\ ${ }^{2}$ The Vice-Chancellor's Office, Universidade Pedagogica , \\ Mozambique \\ ${ }^{3}$ Association for the Development of Veterinary Sciences \\ (ACIVET), Faculty of Veterinary Medicine, Lisbon University, \\ Portugal
}

Correspondence: Tito Fernandes, Association for Development of Veterinary Sciences (ACIVET), Faculty of Veterinary Medicine, Lisbon University, I 300-477 Lisboa, Portugal, Tel +351919927930, Email profcattitofernandes@gmail.com

Received: November 28, 2017 | Published: March 13, 2018

\section{Introduction}

Human nutrition begins with milk. Fermented milk products have been recognized as healthy foods since ancient times. Fermentation processes and products are believed to have been developed 9000 years ago in order to preserve food for times of deficiency, improve flavour, and reduce poisonous effects. Recommendations for the consumption of certain nutritious foods date back to the Hippocratic Corpus of Ancient Greece ${ }^{1}$ are still praiseworthy. Increasing attention has been paid to the importance of nutrition early in life, including the foetal milieu. Thousands of different fermented foods and beverages are still unknown outside the native area in which they have been produced for centuries, many going back even before recorded history. ${ }^{2}$ Fermented foods and beverages pass through a process of lacto fermentation in which natural bacteria or yeasts feed on the sugar and starch in the food creating lactic acid.

The list of fermented products is extremely vast and the diversity derives from the heterogeneity of traditions found in the world, the cultural preference, different geographical areas where they are produced and the staple and/or by-products used for fermentation. The most popular involve beverages such as wine, beer, cider and foods such as yoghurt, cheese, soya, beans, fish, meat, cabbages, among others. In many instances, it is highly likely that the methods of production were unknown and came about by chance, and were passed down by cultural traditional values to subsequent generations. ${ }^{3}$ Modern food is submitted to many processing methods such as pasteurization, affecting its nutritional value by reducing vitamins, fibre, minerals, essential fatty acids and amino acids. Food security can be enhanced in rural areas with fermented products, generating income in a small-scale family farm in developing countries. ${ }^{4}$ The importance of fermentation is reflected by the amount and variety of foods and beverages traded not only for the benefits on nutrition and health-promoting effects but also for preservation, safety, and their peculiar appreciated sensory attributes. ${ }^{5}$ The exploration of the microbial communities and enzymes of fermented products has been extensively reviewed. ${ }^{6}$ At the genus level, Lactobacillusis usually the most abundant genus, followed by Lactococcus, Enterococcus, Vibrio,
Weissella, Pediococcus, Enterobacter, Salinivibrio, Acinetobacter, Macrococcus, Kluyvera and Clostridium.

\section{Fermented products in different guidelines}

Serving small amounts of fermented, cultured or raw food with each meal is pivotal in creating a well-balanced nutritious meal. The Greeks pickle olives, Germans turn cabbages into appetizing sauerkraut and the Japanese transform green, immature plums into the tasty medicinal pickled plums. Food and Nutrition Guidelines for Healthy Children and Young People (aged 2-18 years) exist in many developed countries. ${ }^{7}$ Evaluating dietary guidelines among various countries can help identify their strengths and limitations, yet such assessments are lacking due to the complexity involved. Trying to compare qualitatively current population-level dietary recommendations and pictorial food guides issued by government or nutrition agencies across nutrition transition stages (early, ongoing, and transitioned) is almost an impossible task. ${ }^{8}$ Health practitioners will benefit from recognising and understanding different cultural practices and beliefs in relation to health, food and nutrition. Supporting, valuing and celebrating diverse cultural practices and traditions is recommended for organisations that deal with different ethnical groups. ${ }^{9}$ The recommendations for consumption of foods or dietary practices within main categories of food groups, nutrients, or beverages, including lifestyle, nutritional, cooking, or eating habits is an intricate process. Milk or fermented milk products may block the absorption of phytonutrients, some of which are considered essential. Just because a food is fermented, does not mean it necessarily still has live cultures but the benefits based on the pro biotic activity would require a product that is live. Fermented foods are high in antioxidants, pre-and pro biotics and its beneficial bacteria can help prevent illness. It balances hormones, strengthens the immune and digestive systems and helps manage chronic inflammation.

\section{Microbiota}

Most gut bacteria are beneficial, aiding food digestion, producing vitamins, and protecting against harmful bacteria. However, if left unchecked, harmful bacteria can excrete dangerous metabolites or 
disturb a balance in metabolites that can affect the gut and the rest of the body, including the brain. Many processes in the gut and nervous systems may be interconnected. Previous research has suggested that some, perhaps many, children with autism have abnormal communities of digestive bacteria in their intestines. ${ }^{10}$ Some of these studies have associated specific types of gut bacteria with moresevere autism symptoms. These bacteria include Clostridia and other species produce prop ionic acid and related fatty acids by antibioticresistant gut bacteria that can increase the risk of autism or worsen its symptoms. They tend to overgrow in the gut following a course of antibiotics that wipes out other intestinal bacteria causing intestinal inflammation. ${ }^{11,12}$ The cause of autism is not yet understood, and there is no cure for the disorder. However, certain therapies are associated with an improvement of autistic behaviours. ${ }^{13}$

\section{Offering children fermented foods}

Eating a diet of wholesome foods encourages a wholesome relationship with the world we live in. A number of traditional and semi-traditional foods continue to be an important part of the diet for many communities in the world and for health practitioners; clinical and professional competence cannot be separated from cultural competence. The interest in fermented foods for babies and children stems from the knowledge of the role of gut flora and the human micro biome on general health. Good examples of fermented foods for babies include fruit (e.g. coconut) kefir, fermented sweet potatoes, applesauce and sauerkraut. Offering children a variety of foods helps to broaden their palates, but "sour" is an important flavor that is not usually given to little ones in western cultures. Traditionally fermented foods are a wonderful way to introduce sour flavors. Some children object not to the flavor of fermented foods, but, rather, to the texture. Whole vegetable ferments also allow children to grasp, gnaw on and dip their fermented vegetables into various sauces or mayonnaises, further adding to the appeal.

Many children consume highly processed foods that are overwhelmingly sweet and salty. Giving fermented foods to children from an early age can actually lessen their desire to over consume sweet foods. Children will either love it or hate it, so one has to keep at it trying to provide a healthy balance of food sources.Breast milk contains healthy fat (more than 500 different fatty acids have been identified in cow's milk lipids ${ }^{14}$ ) and bacteria, but the food most commonly fed babies lacks both. Fermented foods really are "food as medicine". They these products aid digestion, support immunity, and actually increase the nutritional content of foods. Once a baby has begun solids foods, they will benefit from the inclusion of small amounts of fermented foods right away. Dipping a spoon or finger in the juice of sauerkraut or other fermented vegetable and letting the baby taste it is a way to adapt the eating habits.

The benefits of fermented foods are not only their pro biotic qualities but also allowing the child to experience and become accustomed to sour flavors. Actually there are on the market vast amount of kid friendly fermented foods although not mentioned on dietary guidelines. Children with neurobehavioral disorders like ADHD (Attention-deficit/hyperactivity disorder), Aspergers syndrome (a milder form of autism) and Processing Disorders often have stomach and digestive upset, often referred to as leaky gut. Symptoms related to these behavioural, social and even academic issues can improve through dietary changes that include pro biotic foods. Mushroom biomass supplements, pro biotic and fermented foods may play a role in impairing these disorders..$^{15}$ The recorded benefits can be either from the live pro biotics or from the enhanced digestibility from fermented foods. Fermented foods restore gut health more effectively than taking a pro biotic supplement. Ideally, these foods should be incorporated with every meal to restore digestive balance and improve emotional well-being and behaviour. However, the scientific support for current Dietary Recommendations, non-existent for fermented foods, is still generally weak for several reasons: limited number, statistical power and scope of intervention studies; limited efficacy of attempted interventions; and lack of generalizability of studies of feeding behaviours at younger ages.

\section{Conclusions and recommendations}

The present global regulatory framework for fermented foods and beverages is confusing and limiting. Foods prepared by fermentation will increase in amount and use as they contribute to the diversity of gut microbiota and indirect impact on mental health and other disorders. The knowledge about the microbial ecology of food and beverage ecosystems is essential in order to understand the production process. Many challenges still remain regarding the establishment of dietary guidelines integrating education, agriculture, health, environment and industry. Supporting children in creating a healthy relationship with nutritious foods is one of the most important relationships a child can make. It is the foundation from which they build the rest of their lives.

\section{Acknowledgment}

None.

\section{Conflict of interest}

None.

\section{References}

1. Lloyd GER, Chadwick J, Mann WN. Regimen for Health. Hippocratic Writings; Penguin Books Ltd.: London, UK, 1983;154-196.

2. Marshall E, Mejia D, editors. Food and Agriculture Organization (FAO) Traditional Fermented Food and Beverages for Improved Livelihoods; FAO: Rome, Italy, 2012.

3. Fijan S. Microorganisms with claimed probiotic properties: An overview of recent literature. Int J Environ Res Public Health. 2014;11(5):47454767.

4. Motarjemi Y. The impact of small-scale fermentation technology on food safety in developing countries. Int J Food Microbiol. 2000;75(3):213229.

5. Food and Agriculture Organization (FAO). Human Nutrition in the Developing World; FAO:Rome, Italy, 1997;p.508.

6. Wood BJB. Microbiology of Fermented Foods, $2^{\text {nd }}$ ed; Thomson Science: Department of Bioscience and Biotechnology, University of Strathclyde, London, United Kingdom,1998;1;1-19.

7. Ministry of Health. Food and Nutrition Guidelines for Healthy Children and Young People (Aged 2-18 years): A background paper. Partial revision, 2012. Wellington: New Zealand.

8. Bell V, Ferrão J, Fernandes TH. Nutritional Guidelines and Fermented Food Frameworks. Foods. 2017;6(8); E65.

9. Parackal S, Ameratunga S, Tin Tin S, et al. The health and wellbeing of secondary school students in New Zealand: Results for Chinese, Indian and other Asian students. 2011; Auckland: The University of Auckland, New Zealand.

10. van De Sande MMH, van Buul VJ, et al. Autism and nutrition: the role of the gut-brain axis. Nutr Res Rev. 2014;2(2);199-214.

11. Slattery J, MacFabe DF, Frye RE. The Significance of the Enteric 
Microbiome on the Development of Childhood Disease: A Review of Prebiotic and Probiotic Therapies in Disorders of Childhood. Clin Med Insights Pediatr. 2016;10:91-107.

12. Frye RE, Rose S, Chacko J, et al. Modulation of mitochondrial function by the microbiome metabolite propionic acid in autism and control cell lines. Transl Psychiatry. 2016;6(10):e927.

13. Kidd PM. An Approach to the Nutritional Management of Autism.
Alternative Therapies. 2003;9(5):22-31

14. Jensen RG. Composition of bovine milk lipids. Journal of the American Oil Chemists Society. 1973;50(6):186-192.

15. Ferrão J, Bell V, Calabrese V, et al. Impact of Mushroom Nutrition on Microbiota and Potential for Preventative Health. Journal of Food and Nutrition Research. 2017;5(4):226-233. 